\title{
Stage-specific embryonic antigen-4 is expressed in basaloid lung cancer and associated with poor prognosis
}

\author{
Sandra Gottschling*, Katrin Jensen* , Arne Warth`, Felix J.F. Herth ${ }^{+}$, \\ Michael Thomas*, Philipp A. Schnabel ${ }^{*}$ and Esther Herpel"
}

ABSTRACT: Basaloid carcinoma represents a rare variant of nonsmall cell lung cancer (NSCLC), which has shown a poor prognosis in a number of studies. Although it is considered to derive from a pluri- or multipotent pulmonary stem cells, little is known about the expression and clinical significance of stem cell antigens in this variant.

Stage-specific embryonic antigen-4 (SSEA-4) was analysed by immunohistochemistry in 38 patients with resected early-stage basaloid NSCLC who had a median follow-up of 72.9 months. The expression of SSEA-4 was related to clinico-pathological characteristics, to the expression of the adult stem cell antigens CD117, CD133 and breast cancer resistance protein 1 (BCRP1), and to prognosis.

SSEA-4 was positive in $37 \%$ of the specimens and showed no association with clinicopathological characteristics or the expression of adult stem cell antigens. Cox proportional hazards regression analysis revealed a 6.0 -fold increased risk of relapse $(p=0.001)$ and a 4.2-fold increased risk of disease-related mortality $(p=0.017)$ in SSEA-4-positive patients, while SSEA-4negative patients showed a prognosis comparable with that of other early-stage NSCLC.

SSEA-4 is expressed in a fraction of basaloid NSCLC and is associated with poor prognosis.

KEYWORDS: Basaloid nonsmall cell lung cancer, biomarker, prognosis, stage-specific embryonic antigen-4

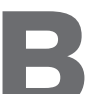
asaloid lung cancer is a rare variant of pulmonary squamous cell carcinoma (SCC) or large cell carcinoma and accounts for $4.8-6.3 \%$ of all nonsmall cell lung cancer (NSCLC) cases [1, 2]. In contrast to overall NSCLC, which has a 5-yr survival rate of, on average, $40-60 \%$ and a median survival time of $\sim 50$ months in stage I/II disease, analyses of two European collectives comprising 38 and 90 patients with basaloid NSCLC revealed a significantly lower 5-yr survival rate of $10-27 \%$ and a shorter median survival time of 20-29 months [1, 3-6]. The basal cell phenotype of these carcinomas and their co-occurrence with virtually all histological subtypes of NSCLC soon led to the assumption that this variant derives from a pluri- or multipotent pulmonary stem cell $[1,5,7]$. In recent years, several in vitro and in vivo studies indicated the presence of stem-like cells in NSCLC. Those were characterised by: the expression of stem cell antigens, such as CD117, CD133 and/or breast cancer resistance protein 1 (BCRP1); the ability to regenerate the primary tumour; an increased metastatic capability; and drug resistance [8-10].
Previous studies in early-stage disease indicated that expression of CD133 and BRCP1 predicts an increased risk of relapse and disease-related mortality after pulmonary resection [11, 12]. In contrast to adult stem cell antigens, the knowledge about the expression and significance of embryonic stem cell (ESC) antigens in lung cancer is scant. The markers most frequently used to identify ESC are the glycolipid carbohydrate epitopes stagespecific embryonic antigen (SSEA)-3 and -4 and the transcription factor Oct-4 [13]. Recent studies demonstrated expression of Oct-4 in a subset of NSCLC cells with stem cell properties and an unfavourable prognostic significance of this antigen in adenocarcinomas with lepidic growth pattern [14-17]. In contrast, SSEA-4, which characterises very early ESCs, has yet been analysed in teratocarcinomas, testicular germ cell tumours and a single study of ovarian cancer, but not in lung cancer [18-20]. Analyses of the expression of stem cell antigens are lacking so far, particularly in basaloid NSCLC, which is predominantly suggested to have an association with pulmonary stem cells. In our study, we analysed tumour

\section{AFFLLIATIONS}

*Dept of Thoracic Oncology,

Thoraxklinik/University of Heidelberg, \#Institute of Medical Biometry and Informatics, University of Heidelberg, "Institute of Pathology, University of Heidelberg, and

${ }^{+}$Dept of Pneumology \& Critical Care Medicine, Thoraxklinik/University of Heidelberg, Heidelberg, Germany.

CORRESPONDENCE

S. Gottschling

Dept of Thoracic Oncology

Thoraxklinik/University of Heidelberg

Amalienstr. 5

69126 Heidelberg

Germany

E-mail: sandra.gottschling@

thoraxklinik-heidelberg.de

Received:

Dec 232011

Accepted after revision:

May 302012

First published online:

June 272012

European Respiratory Journal

Print ISSN 0903-1936

Online ISSN 1399-3003 
tissue of 38 completely resected stage I/II patients with basaloid NSCLC for the expression and prognostic significance of SSEA-4. Moreover, the results were related to clinico-pathological characteristics and the expression of the adult stem cell antigens CD117, CD133 and BCRP1.

\section{MATERIALS AND METHODS Patient and sample characteristics}

A total of 38 previously untreated patients with basaloid NSCLC who underwent complete pulmonary resection between 2002 and 2009 at the Dept of Thoracic Surgery, Thoraxklinik, University of Heidelberg, Heidelberg, Germany, were analysed. Tissue specimens and follow-up data were obtained from the tissue bank and the lung cancer registry of the Thoraxklinik and the tissue bank of the National Center for Tumour Diseases (NCT), University of Heidelberg. Patients had given informed consent following the 2000 revision of the guidelines of the Declaration of Helsinki and the local Ethics Committee of the Medical Faculty Heidelberg. Pre-operative and follow-up assessments were performed according to the guidelines of the German Respiratory Society last published in 2010 [21]. The stage was determined according to the seventh edition of the TNM classification of malignant tumours [3]. Detailed patient characteristics are given in table 1.

\section{Histological classification}

The histological classification of a basaloid NSCLC variant was based on the criteria published in the 2004 revision of the World Health Organization classification of tumours and included: 1) solid lobular or anastomotic trabecular pattern growing invasively in a finger-like fashion from the bronchial and/or glandular duct lining; 2) small cuboidal to fusiform cells of 12$15 \mu \mathrm{m}$ mean diameter with scant, but visible cytoplasm, and moderately hyperchromatic nuclei without nuclear moulding or prominent nucleoli; 3) peripheral palisading with radially arranged cells at the periphery of the tumour lobules; and 4) a mitotic index of $\geqslant 15-44$ mitoses per 10 high-power fields [22]. Haematoxylin-stained tissue sections were used for histological classification and grading. The diagnosis of a squamous variant was based on the presence of intercellular bridges or individual cell keratinisation within the basal cell component in $<50 \%$ of the tumour area. Additionally, the expression of the neuroendocrine (NE) markers neuron-specific enolase, synaptophysin, chromogranin A, CD56 and the high molecular weight cytokeratins (HMWCKs) 1, 5, 10 and 14 was analysed to exclude large cell neuroendocrine or small cell carcinomas. The diagnosis of a basaloid carcinoma was maintained when HMWCKs were expressed and clear-cut staining for at least one specific NE marker was lacking.

\section{Immunohistochemical and immunocytochemical analyses}

Tissue microarrays (TMA) of formalin-fixed, paraffin-embedded tissue derived from pulmonary resections were prepared by the tissue bank of the NCT, as previously described [23]. For each patient, two 1.2-mm diameter tissue cores each of the tumour centre, the invasion front and histologically normal lung tissue were spotted. In half of the cases, additionally, whole tissue sections were prepared. $2-\mu \mathrm{m}$ sections of the TMA or the whole tissue were deparaffinised with xylene and rehydrated in graded alcohol series. For antigen retrieval, the sections were boiled in target retrieval buffer $\mathrm{pH} 6$ (Dako, Glostrup, Denmark) for
15 min. Sections for CD133 and BCRP1 staining were additionally blocked by avidin-biotin treatment. Subsequent steps were performed in an immunostaining device (Autostainer; Dako). Briefly, the sections were incubated with the primary antibody for $30 \mathrm{~min}$, washed with PBS/Tween 20, incubated with the secondary antibody for $20 \mathrm{~min}$ and washed again. Endogenous peroxidase was blocked by incubation with peroxidase-blocking solution (Dako) for $5 \mathrm{~min}$. Detection was based on the avidinbiotin peroxidase principle using AEC as chromogen (Dako REAL Detection System Peroxidase/AEC, Rabbit/Mouse). The sections were counterstained with Mayer's haematoxylin for $5 \mathrm{~min}$ and mounted with coverslips in Aquatex mounting medium (Merck KGaA, Darmstadt, Germany). The following primary antibodies, clones and dilutions were used: SSEA-4 (clone MC-813-70, 1:100; Millipore, Billerica, MA, USA), CD117 (polyclonal, 1:50, Dako), CD133 (polyclonal (ab19898), 1:100; Abcam plc, Cambridge, MA, USA), BCRP1 (clone BXP-21, 1:100; Abcam), Ki67 (clone MiB1, 1:200; Dako), CD56 (clone 1B6, 1:50; Novocastra, A. Menarini Diagnostics Deutschland, Berlin, Germany), synaptophysin (clone 27G12, 1:400; Novocastra), neuron-specific enolase (clone MIG-N3, 1:10,000; DCS Innovative Diagnostik-Systeme Dr. Christian Sartori $\mathrm{GmbH} \& \mathrm{Co}$. KG, Hamburg, Germany), synaptophysin (clone LK2H10, 1:10; Abcam) and HMWCK (clone 34ßE12, 1:100, Abcam) recognising cytokeratin (CK) 1, 5, 10 and 14. For negative controls, the primary antibody was omitted. Teratocarcinoma tissue derived from the tissue bank of the NCT was used as a positive control for $S S E A-4$. For analysis of SSEA-4 expression in nontumoral conditions, lung tissue of healthy subjects with spontaneous pneumothorax was used and provided by the tissue bank of the NCT. The analysis was performed by two independent observers (E. Herpel and P.A. Schnabel). Samples were considered positive, if either dispersed expression or at least one focus with distinct staining was present. The TMA were scanned at $400 \times$ magnification using Aperio ImageScope v10.1.3.2028 software (Aperio Technologies Inc., Vista, CA, USA).

The human embryonic carcinoma cell line NTERA-2 (Deutsche Sammlung für Mikroorganismen und Zellkulturen $\mathrm{GmbH}$, Braunschweig, Germany) and the basaloid NSCLC cell line $2427 \mathrm{~T}$ were cultured as indicated by the supplier or as previously published, respectively [24, 25]. For immunocytochemical analyses, cytospins of each 1 million NTERA-2 or $2427 \mathrm{~T}$ cells were prepared in a Shandon CytoSpin III cytocentrifuge (Fisher Scientific $\mathrm{GmbH}$, Schwerte, Germany). The cells were fixed with neutral buffered $10 \%$ formalin for $5 \mathrm{~min}$, boiled in target retrieval buffer $\mathrm{pH} 6$ (Dako) for $15 \mathrm{~min}$ and permeabilised with Triton X-100 for $10 \mathrm{~min}$. All subsequent steps were performed as described above.

\section{Statistical analyses}

The follow-up was defined as the Kaplan-Meier estimate with reversed status indicator. Death censored the true but unknown observation time, censoring was interpreted as end-point. Thus, the unobservable follow-up time of a deceased patient was interpreted as the follow-up time that potentially would have been obtained if the patient had not died. Survival time was determined from the date of first diagnosis until last follow-up or reported death. Nondiseaserelated death was censored. The disease-free survival was determined from the date of first diagnosis until diagnosis of 


\begin{tabular}{|c|c|c|c|c|}
\hline & Total & SSEA-4 positive & SSEA-4 negative & $\mathrm{p}$-value \\
\hline \multicolumn{5}{|l|}{ Sex } \\
\hline Female & $7(18)$ & $2(14)$ & $5(21)$ & 0.615 \\
\hline Male & $31(82)$ & $12(86)$ & $19(79)$ & \\
\hline \multicolumn{5}{|l|}{$\mathrm{ECOG}^{\#}$} \\
\hline 0 & $28(74)$ & $11(79)$ & $17(71)$ & 0.601 \\
\hline$\geqslant 1$ & $10(26)$ & $3(21)$ & 7 (29) & \\
\hline \multicolumn{5}{|l|}{ UICC stage } \\
\hline $\mathrm{IA}$ & $6(16)$ & $2(14)$ & $4(17)$ & 0.899 \\
\hline IB & $13(34)$ & $4(29)$ & $9(37)$ & \\
\hline T2 & $28(74)$ & $10(71)$ & $18(75)$ & \\
\hline \multicolumn{5}{|l|}{ pN } \\
\hline No & $21(55)$ & $7(50 \%)$ & $14(58)$ & 0.618 \\
\hline $\mathrm{N} 1$ & $17(45)$ & $7(50 \%)$ & $10(42)$ & \\
\hline \multicolumn{5}{|l|}{ Type of resection } \\
\hline Pneumonectomy & $6(16)$ & $4(29)$ & $2(8)$ & 0.247 \\
\hline Bilobectomy & $4(10)$ & $1(7)$ & $3(13)$ & \\
\hline Lobectomy & $28(74)$ & $9(64)$ & $19(79)$ & \\
\hline \multicolumn{5}{|l|}{ Relapse \% } \\
\hline Total & $16(42)$ & $11(79)$ & $5(21)$ & 0.000 \\
\hline Local & $1(3)$ & $0(0)$ & $1(4)$ & NA \\
\hline $1-y r$ & $91.7(82.7-100)$ & $85.1(66.0-100)$ & $95.7(87.3-100)$ & NA \\
\hline $2-y r$ & $83.0(70.6-95.5)$ & $69.6(44.7-94.6)$ & $90.9(78.7-100)$ & \\
\hline $5-y r$ & $61.9(44.4-79.4)$ & $35.4(8.1-62.7)$ & $80.1(64.4-97.8)$ & \\
\hline Follow-up months & $72.9(42.7-91.5)$ & $91.4(42.7-96.3)$ & $70.2(37.5-84.2)$ & NA \\
\hline
\end{tabular}

Data are presented as $n, n(\%)$, mean \pm SEM or median $(95 \% \mathrm{CI})$, unless otherwise stated. SSEA-4: stage-specific embryonic antigen-4; ECOG: Eastern Cooperative Oncology Group; UICC: Union Internationale Contre le Cancer; NR: not reached; NA: not applicable. p-values in bold are statistically significant. ${ }^{\#}$ : ECOG 0 is a patient without physical restriction and $E C O G \geqslant 1$ is a patient with increasing physical restriction. Bold indicates statistical significance.

relapse or disease-related death. Survival times were analysed using the Kaplan-Meier method and the log-rank test. For multivariate analysis the Chi-squared test and Wilcoxon rank sum test were used to evaluate the difference between groups. A p-value $<0.05$ was considered statistically significant. The statistical analyses were performed using $\mathrm{SAS} \otimes$ version 9.2 (SAS Institute, Cary, NC, USA).

\section{RESULTS}

\section{Expression of SSEA-4 in basaloid NSCLC}

Tissue specimens of 38 patients with stage I/II basaloid NSCLC were analysed for the expression of SSEA-4. The collective contained 24 pure basaloid carcinomas, 13 basaloid carcinomas with a SCC component and one basaloid carcinoma with an adenocarcinoma component. In all specimens, the basaloid component comprised $\geqslant 60 \%$ of the tumour bulk. SSEA-4 showed diffuse membranous/cytoplasmic expression in $14(37 \%)$ out of 38 of the specimens (figs $1 \mathrm{a}$ and $2 \mathrm{a}$ ). The positive control, a teratocarcinoma, likewise showed diffuse cytoplasmic/membranous and, in some cells, also showed nuclear staining (fig. 2b). In the NSCLC specimens, positive staining was restricted to the basaloid component, while the well differentiated adenocarcinoma (AC) or SCC components were negative for SSEA-4 (fig. $2 \mathrm{c}$ and $2 \mathrm{~d}$ ). The alveolar 

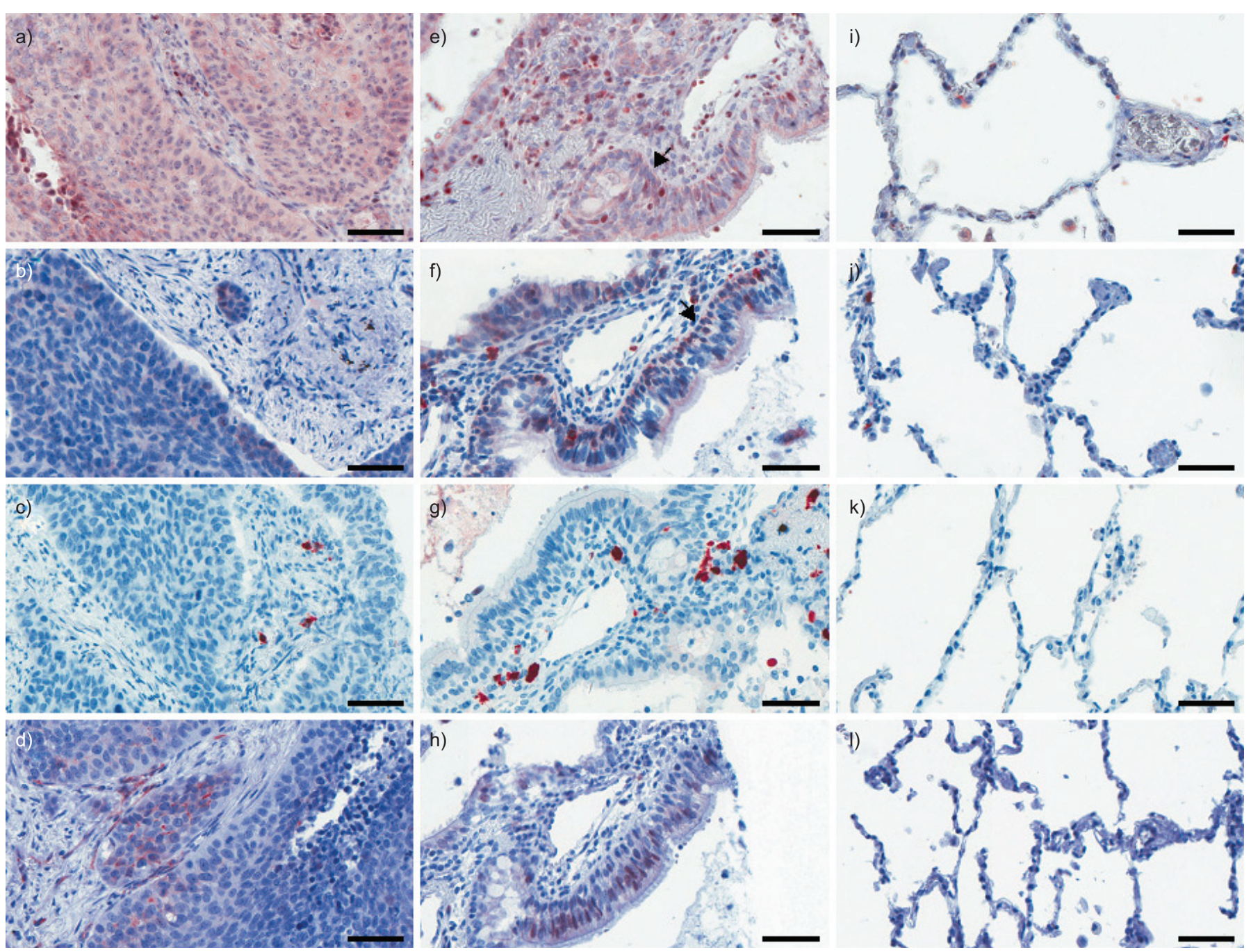

FIGURE 1. a-d) Basaloid nonsmall cell lung cancer, e-h) tumour-associated tracheobronchial epithelium, and i-l) tumour-associated alveolar epithelium specimens analysed for a, e, i) stage-specific embryonic antigen-4 (SSEA-4), b, f, j) CD117, c, g, k) CD133 and d, h, I) breast cancer resistance protein 1 (BCRP1). Scale bars=50 $\mu \mathrm{m}$.

epithelium of patients and healthy subjects was negative for SSEA-4 (figs $1 \mathrm{i}$ and 2e), whereas the tracheobronchial epithelium displayed membranous/cytoplasmic staining of the basal cells and cytoplasmic accumulation at the apical cell pole of ciliated cells (figs 1e and 2f). All specimens displayed reactivity for HMWCK, while clear-cut expression of specific NE markers was absent (fig. 3). Remarkably, the basaloid NSCLC cell line $2427 \mathrm{~T}$ which shows properties related to stem cells, i.e. high tumorigenicity in the animal model and reassembling of the original tumour histomorphology, also displayed expression of SSEA-4. Similarly, the positive control, the embryonic carcinoma cell line NTERA-2 showed distinct membranous/cytoplasmic staining (fig. 4).

\section{SSEA-4, clinico-pathological characteristics and adult stem cell antigens}

The expression of SSEA-4 showed no association with clinicopathological characteristics or the expression of adult stem cell antigens (tables 1 and 2). CD117 was positive in $47 \%, B C R P 1$ in $50 \%$ and CD133 in 5\% of the tumours. CD117 showed diffuse membranous and/or cytoplasmic staining (fig. $1 b, f$ and $j$ ), while BCRP1 displayed additionally nuclear staining and predominantly focal expression (fig. $1 \mathrm{~d}, \mathrm{~h}$ and $\mathrm{l}$ ). The alveolar epithelium of patients was negative for adult stem cell antigens (fig. 1i-1), whereas the tracheobronchial epithelium showed cytoplasmic expression of CD117 in the basal cells and in some ciliated cells and nuclear expression of BCRP1 in the majority of ciliated cells (fig. 1e-h).

\section{Prognostic significance of SSEA-4}

Within the median follow-up time of 91.4 months for SSEA-4positive and 70.2 months for SSEA-4-negative patients, significantly more individuals with SSEA-4-positive than SSEA-4negative tumours experienced relapse $(79 \%$ versus $21 \%$, $\mathrm{p}=0.0005$ ). All but one relapse were distant relapses (table 1). The 5-yr survival rate of SSEA-4-positive patients was 35\% and that of SSEA-4-negative patients $80 \%$. Cox proportional regression analysis revealed a 6.0 -fold increased risk of relapse (hazard ratio (HR) 6.0, 95\% CI 2.1-17.4; $\mathrm{p}=0.001$ ) and a 4.2fold increased risk of disease-related mortality (HR 4.2, 95\% CI 1.3-13.7; $\mathrm{p}=0.017$ ) in patients with SSEA-4-positive tumours (figs $5 \mathrm{a}$ and $5 \mathrm{~b}$ ). These patients showed a median disease-free 

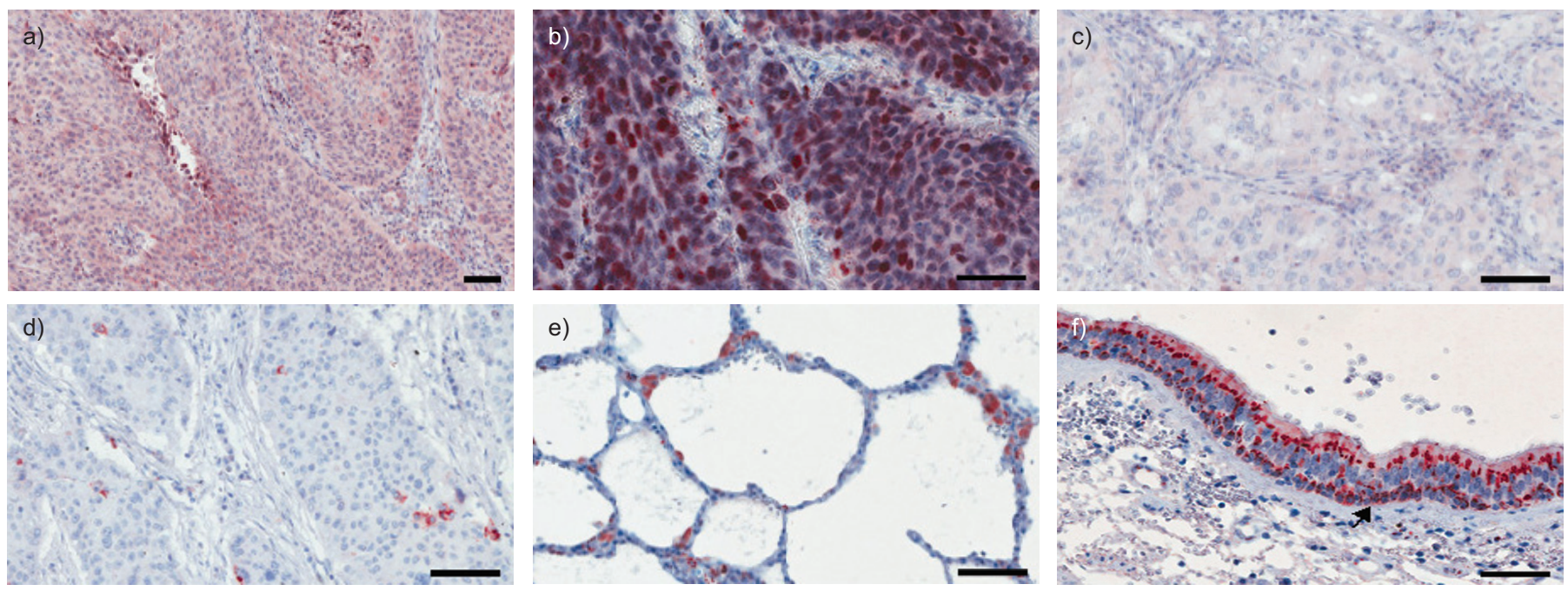

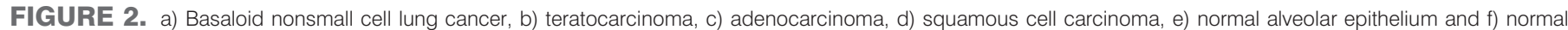
tracheobronchial epithelium specimens analysed for stage-specific embryonic antigen-4. a) Scale bar $=100 \mu \mathrm{m}$ and b-f) scale bars $=50 \mu \mathrm{m}$

survival of 11.1 months and median overall survival of 45.0 months, while the median disease-free and overall survival of patients with SSEA-4-negative tumours were not reached.

\section{DISCUSSION}

Basaloid lung cancer represents a rare variant of NSCLC and showed an unfavourable prognosis in a number of studies $[1,5$, 6]. BRAMBILLA et al. [5], who were the first to describe this variant, speculated as early as 1992 that basaloid NSCLC might derive from a pluri- or multipotent pulmonary stem cell. Although a few studies indicated the expression and unfavourable prognostic significance of embryonic and adult stem cell antigens, such as SSEA-1, Oct-4, CD133 and BCRP1 in NSCLC, analyses particularly in basaloid lung cancer are lacking so far $[11,12,16$, $17,26,27]$. In our study analysing 38 patients with early-stage basaloid NSCLC, we found expression of the early ESC antigen SSEA-4 in $37 \%$ of the specimens and an association with a 6.0-fold increased risk of relapse and a 4.2-fold increased risk of disease-related mortality. While patients with SSEA-4-negative tumours showed a relapse rate of $21 \%$ and a 5 -yr survival rate of $80 \%$, patients with SSEA-4-positive tumours were recurrent in $79 \%$ of the cases and showed a 5 -yr survival rate of $35 \%$. Without stratification for SSEA-4, the relapse rate (42\%) and 5-yr survival rate $(62 \%)$ of the entire collective was similar to that reported for overall early-stage NSCLC or poorly differentiated SCC (PDSC) [1-6]. This is in contrast to the results of Brambilla and co-workers $[1,5,6]$, who reported a significantly worse outcome of basaloid carcinomas as compared with other NSCLC or PDSC: While the latter displayed a 5-yr survival rate of $44 \%$ and $47-55 \%$ in stage I/II disease, respectively, the 5-yr survival rate of early-stage basaloid NSCLC was 10-27\% [1, 5, 6]. Conversely, previous studies of KIM et al. [2] and WANG et al. [28] in two Asian collectives likewise demonstrated comparable relapse rates $(33-55 \%)$ and 5 -yr survival rate $(50-57 \%)$ for
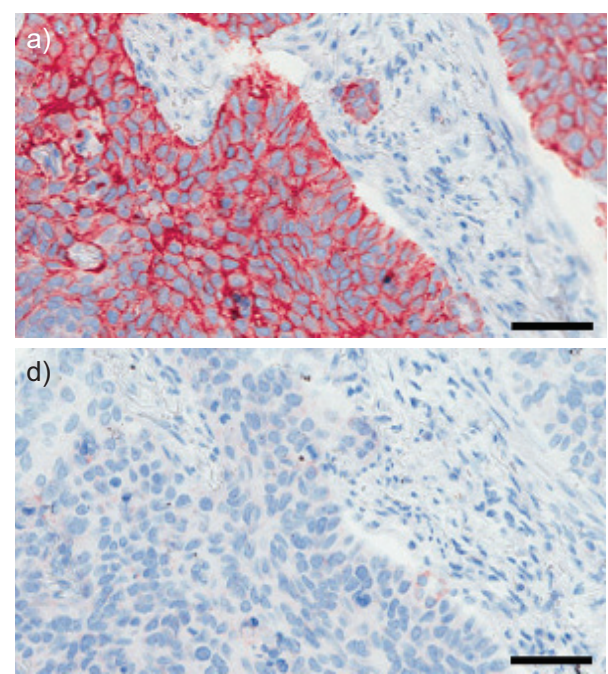
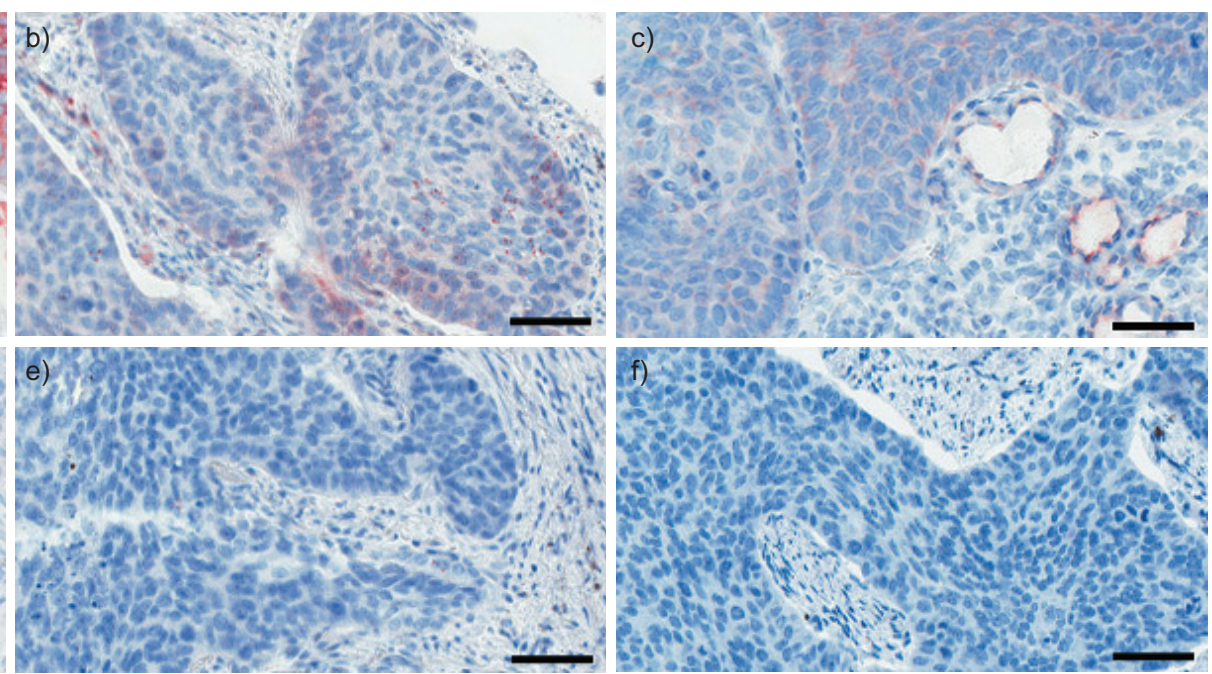

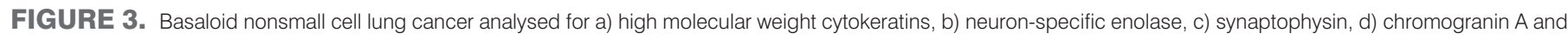
e) CD56, and f) negative control. Scale bars $=50 \mu \mathrm{m}$. 

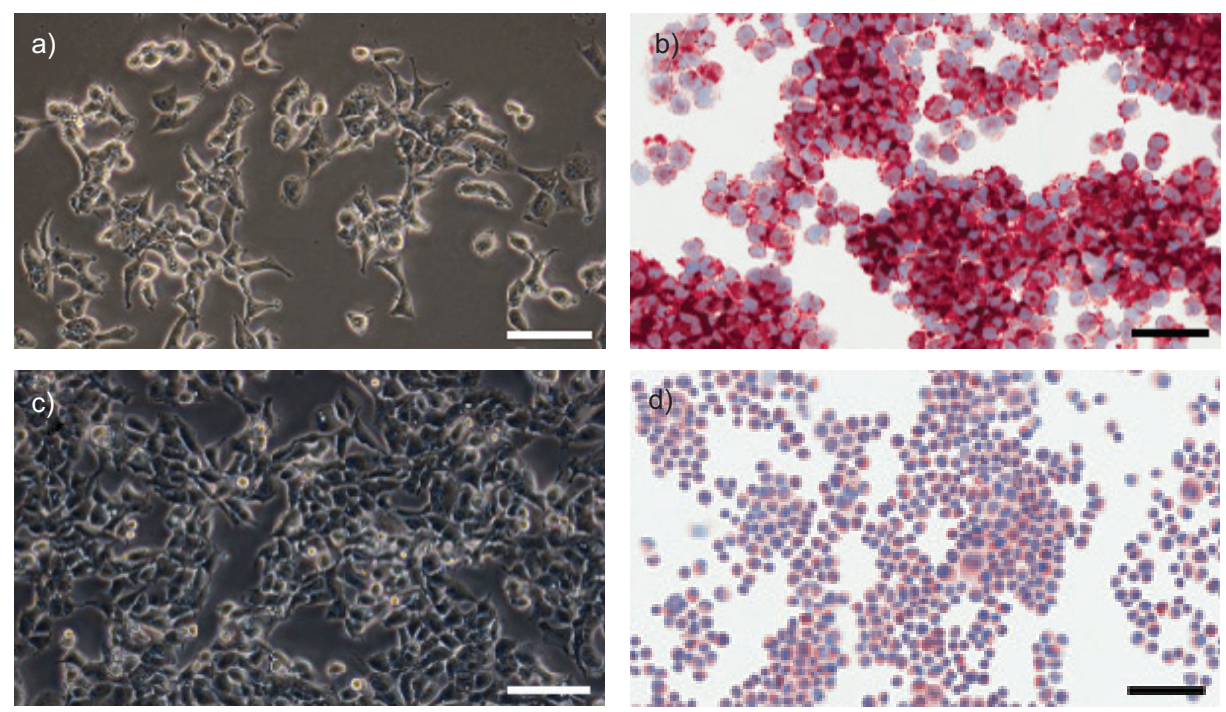

FIGURE 4. Phase contrast microscopy of a) NTERA-2 and c) $2427 T$ and stage-specific embryonic antigen-4 (SSEA-4) analysed b) NTERA-2 and d) $2427 T$. Scale bars $=100 \mu \mathrm{m}$

basaloid carcinoma and PDSC. The results of our study might provide one of the reasons for the inconsistent findings of previous studies, as a random accumulation of patients with SSEA-4-positive tumours might result in worse prognosis of the entire study population with basaloid NSCLC. However, several other factors have to be considered for these discrepancies between the studies including technical aspects, investigator's variability in the identification of basaloid NSCLC, changes of the staging system, differences in the surgical procedure and post-operative treatment phase, differences in the interval and type of clinical assessments, as well as differences of the number of analysed subjects, the follow-up time and clinico-pathological characteristics of the study population. Moreover, the better treatment options for relapsed patients since 2000 might have levelled discrete survival differences in the more recent studies of KIM et al. [2], WANG et al. [28] and WAECHTER et al. [29].

Besides clinical significance, the expression of the early ESC antigen SSEA-4 in basaloid carcinoma and in basal cells of the tracheobronchial epithelium represents an important

\begin{tabular}{|c|c|c|c|c|}
\hline \multirow[t]{2}{*}{ TABLE 2} & \multicolumn{4}{|c|}{$\begin{array}{l}\text { Expression of stage-specific embryonic } \\
\text { antigen-4 (SSEA-4) and adult stem cell antigens }\end{array}$} \\
\hline & Total & $\begin{array}{l}\text { SSEA-4 } \\
\text { positive }\end{array}$ & $\begin{array}{c}\text { SSEA-4 } \\
\text { negative }\end{array}$ & p-value \\
\hline Samples $\mathrm{n}$ & 38 & 14 & 24 & \\
\hline CD117 & $18(47)$ & $6(43)$ & $12(50)$ & 0.671 \\
\hline CD133 & $3(8)$ & $2(14)$ & $1(4)$ & 0.264 \\
\hline BCRP1 & $19(50)$ & $8(57)$ & $11(46)$ & 0.501 \\
\hline CD117/BCRP1 & $11(29)$ & $3(21)$ & $8(33)$ & 0.435 \\
\hline
\end{tabular}

pathophysiological aspect and supports the stem cell hypothesis of BRAMBILLA et al. [5]. In line with rapid downregulation of this glycolipid antigen in the embryonic carcinoma cell lines 2102Ep , BG01V, NTERA-2 and in blastocyst-derived ESC upon differentiation, we found no expression of SSEA-4 in welldifferentiated tumour components of basaloid NSCLC [30-34]. Moreover, a recently established basaloid NSCLC cell line that shows properties related to stem cells, i.e. high tumorigenicity in the animal model and reassembling of the original tumour histomorphology, also displayed expression of SSEA-4 [25]. However, as defined model systems and conditions for the assessment of multi-lineage differentiation capacity of pulmonary cells are lacking so far, the nature of SSEA-4-positive cells in the tracheobronchial epithelium and in some basaloid NSCLC remains to be elucidated. As for the current study, the results suggest that a part of the basaloid carcinomas retain the embryonic antigen expression of their putative cell of origin, while others lack either any stem-cell antigens or show exclusively expression of adult stem cell markers. It may be speculated whether these carcinomas derive from more committed (stem) cells or undergo differentiation during tumour growth. The lack of expression of CD133 and BCRP1 in the basal cells of the tracheobronchial epithelium, but focal expression of BCRP1 in some SSEA-4-positive tumours suggests the capability of intratumoral differentiation.

As the representativeness of the TMA plays a critical role particularly in focally expressed antigens, such as $B C R P 1$, the TMA was prepared according to recent validation data in lung cancer and mesothelioma that demonstrated that three to four tissue cores per sample are sufficient to produce reliable results [35-37]. Moreover, in half of the cases, the results of the TMA were compared with corresponding whole tissue sections and showed full concordance.

With expression of the early ESC antigen SSEA-4 in basaloid carcinoma and in the basal cells of the tracheobronchial epithelium, the transcription factor and proto-oncogene $c-m y c$ has to be discussed as a potential molecular driver of this 


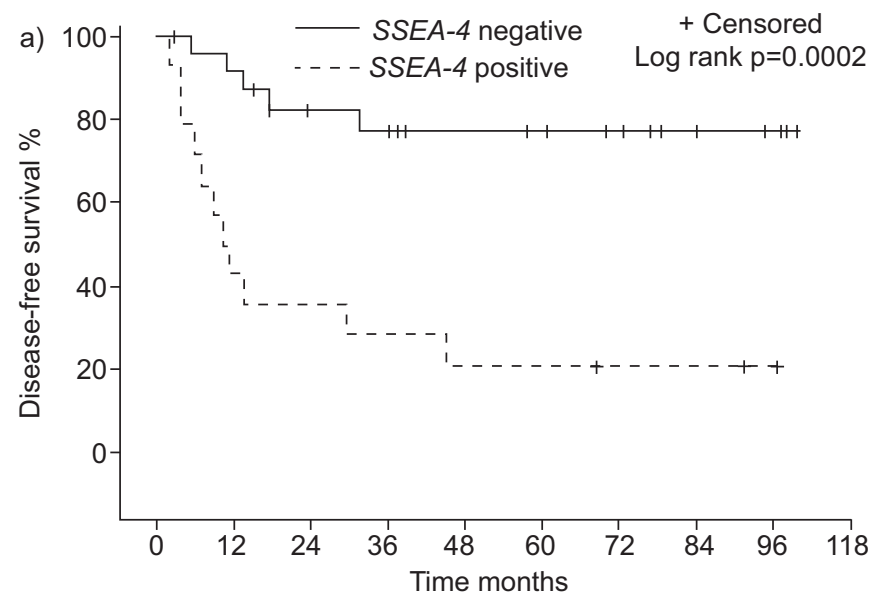

$\begin{array}{cccccccccc}24 & 21 & 16 & 15 & 12 & 11 & 9 & 6 & 3 & 0 \\ 14 & 6 & 5 & 4 & 3 & 3 & 2 & 2 & 1 & 0\end{array}$

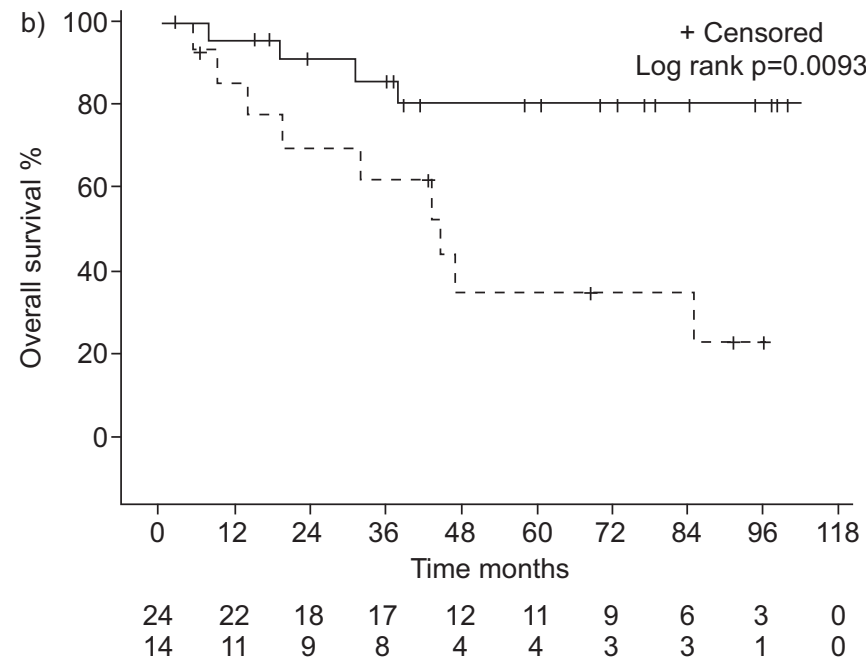

FIGURE 5. a) Disease-free and b) overall survival for stage-specific embryonic antigen-4 (SSEA-4)-positive and -negative patients. Numbers below the graphs represent the actual numbers of SSEA-4-negative (top row) and SSEA-4-positive patients (bottom row).

NSCLC variant. $c-m y c$ represents one of the factors that is crucially involved in the early steps of somatic cell reprogramming and is the centre of a regulatory network that induces an embryonic gene expression profile, albeit with no pluripotency in cancer cells $[38,39]$. Depending on the method, $5.6-80 \%$ of all NSCLCs show an amplification of this gene and $48-58 \%$ an overexpression [40-42]. Remarkably, in normal tracheobronchial epithelium, the highest expression values were reported for basal cells, and tumours arising from these natural progenitor cells might per se display ESC features [42]. To date, no study or subgroup analysis of basaloid lung cancer is known to the authors that would have analysed the status of $c$ $m y c$ or the genetic profile of this variant. Our own comparative genomic hybridisation and multiplex fluorescence in situ hybridisation analysis of two primary tumours and one lymph node metastasis of basaloid NSCLC that was conducted in the context of cell line establishment demonstrated a consistent amplification of $8 \mathrm{q} 24$, the gene locus of $c-m y c$ [25].
Despite this, the presence of tumour cells with ESC characteristics might provide novel therapeutic options and targets for NSCLC: glycolipid antigens, such as SSEA-4, are highly immunogenic and might be suitable for antibody-based therapy approaches, whereas embryonic signalling pathways, such as Wnt/ $\beta$-catenin, hedgehog or notch, could be a target for small molecule inhibitors [43, 44].

\section{Conclusions}

This study demonstrated expression of the ESC antigen SSEA-4 in a fraction of basaloid NSCLC and in the basal cells of the tracheobronchial epithelium. Patients with SSEA-4positive tumours showed a significantly increased risk of relapse and disease-related mortality. With respect to the limited sample size of this study, which precludes a definitive statement, SSEA-4 might represent a promising candidate for the identification of patients with basaloid NSCLC who will benefit from an adjuvant therapy after surgery.

\section{STATEMENT OF INTEREST}

None declared.

\section{ACKNOWLEDGEMENTS}

The authors thank B. Walter (Tissue Bank, National Center for Tumour Diseases, Heidelberg, Germany) for excellent technical assistance.

\section{REFERENCES}

1 Moro-Sibilot D, Lantuejoul S, Diab S, et al. Lung carcinomas with a basaloid pattern: a study of 90 cases focusing on their poor prognosis. Eur Respir J 2008; 31: 854-859.

2 Kim DJ, Kim KD, Shin DH, et al. Basaloid carcinoma of the lung: a really dismal histologic variant? Ann Thorac Surg 2003; 76: 1833-1837.

3 Detterbeck FC, Boffa DJ, Tanoue LT. The new lung cancer staging system. Chest 2009; 136: 260-271.

4 Pfannschmidt J, Muley T, Bülzebruck H, et al. Prognostic assessment after surgical resection for non-small cell lung cancer: experiences in 2083 patients. Lung Cancer 2007; 55: 371-377.

5 Brambilla E, Moro D, Veale D, et al. Basal cell carcinoma (basaloid) carcinoma with separate prognostic significance. Hum Pathol 1992; 23: 993-1003.

6 Moro D, Brichon P-Y, Brambilla E, et al. Basaloid bronchial carcinoma. A histologic group with a poor prognosis. Cancer 1994; 73: 2734-2739.

7 Marci V, Volante M, Cappia S, et al. Basaloid adenocarcinoma. A new variant of pulmonary adenocarcinoma. Virchows Arch 2007; 451: 729-736.

8 Eramo A, Lotti F, Sette G, et al. Identification and expansion of the tumorigenic lung cancer stem cell population. Cell Death Diff 2008; 15: 504-514.

9 Bertolini G, Roz L, Perego P, et al. Highly tumorigenic lung cancer CD133+ cells display stem-like features and are spared by cisplatin treatment. Proc Natl Acad Sci USA 2009; 106: 16281-16286.

10 Kajstura J, Rota M, Hall SR, et al. Evidence for human lung stem cells. NEJM 2011; 364: 1795-1806.

11 Li F, Zeng H, Ying K. The combination of stem cell markers CD133 and ABCG2 predicts relapse in stage I non-small cell lung carcinomas. Med Oncol 2011; 28: 1458-1462.

12 Woo T, Okudela K, Mitsui $\mathrm{H}$, et al. Prognostic value of CD133 expression in stage I lung adenocarcinomas. Int J Clin Exp Pathol 2010; 4: 32-42.

13 The International Stem Cell Initiative. Characterization of human embryonic stem cell lines by the International Stem Cell Initiative. Nature Biotech 2007; 25: 803-816. 
14 Levina V, Marrangoni AM, DeMarco R, et al. Drug-selected human lung cancer stem cells: cytokine network, tumorigenic and metastatic properties. PLoS ONE 2008; 3: e3077.

15 Chen Y-C, Hsu H-S, Chen Y-W, et al. Oct-4 expression maintained cancer stem-like properties in lung cancer-derived CD133-positive cells. PLOS ONE 2008; 3: e2637.

16 Karoubi G, Gugger M, Schmid R, et al. OCT4 expression in human non-small cell lung cancer: implications for therapeutic intervention. Interact Cardiovasc Thorac Surg 2009; 8: 393-397.

17 Zhang X, Han B, Huang J, et al. Prognostic significance of OCT4 expression in adenocarcinoma of the lung. Jpn J Clin Oncol 2010 40: 961-966.

18 Krupnik JG, Damjanov I, Damjanov A, et al. Globo-series carbohydrate antigens are expressed in different forms of human and murine teratocarcinoma-derived cells. Int J Cancer 1994; 59: 692-698.

19 Tokuyama S, Saito S, Takahashi $T$, et al. Immunostaining of stagespecific embryonic antigen-4 in intratubular germ cell neoplasia unclassified and in testicular germ-cell tumors. Oncol Rep 2003; 10 : 1097-1104.

20 Ye F, Li Y, Hu Y, Zhou C, et al. Stage-specific embryonic antigen 4 expression in epithelial ovarian carcinoma. Int J Gyn Cancer 2010; 20: 958-964.

21 Goeckenjan G, Sitter H, Thomas M, et al. Prevention, diagnosis, therapy, and follow-up of lung cancer. Pneumologie 2010; 64: Suppl. 2, e1-e164.

22 Travis WD, Brambilla E, Müller-Hermelink HK, et al. Pathology and Genetics: Tumours of the Lung, Pleura, Thymus and Heart. Lyon, International Agency for Research on Cancer, 2004.

23 Warth A, Muley T, Meister M, et al. The novel histologic IASLC/ ATS/ERS classification system of invasive pulmonary adenocarcinoma is a stage-independent predictor of survival. J Clin Oncol 2012; 30: 1438-1446.

24 Draper JS, Pigott C, Thomson JA, et al. Surface antigens of human embryonic stem cells: changes upon differentiation in culture. $J$ Anat 2002; 200: 249-258.

25 Gottschling S, Jauch A, Kuner R, et al. Establishment and comparative characterization of novel squamous cell non-small cell lung cancer cell lines and their corresponding tumor tissue. Lung Cancer 2012; 75: 45-57.

26 Fukuoka K, Narita N, Saijo N. Increased expression of sialyl Lewis(x) antigen is associated with distant metastasis in lung cancer patients: immunohistochemical study on bronchofiberscopic biopsy specimens. Lung Cancer 1998; 20: 109-116.

27 Ogawa J, Sano A, Inoue $\mathrm{H}$, et al. Expression of Lewis-related antigen and prognosis in stage I non-small cell lung cancer. Ann Thorac Surg 1995; 59: 412-415.

28 Wang LC, Wang L, Kwauk S, et al. Analysis on the clinical features of 22 basaloid squamous cell carcinoma of the lung. J Cardiothoracic Surg 2011; 6: 10.

29 Waechter F, Passweg J, Tamm M, et al. Significant progress in palliative treatment of non-small cell lung cancer in the past decade. Chest 2005; 127: 738-747.
30 Kannagi R, Cochran NA, Ishigami F, et al. Stage-specific embryonic antigens (SSEA-3 and -4) are epitopes of a unique globo-series ganglioside isolated from human teratocarcinoma cells. EMBO 1983; 2: 2355-2361.

31 Josephson R, Ording CJ, Liu Y, et al. Qualification of embryonal carcinoma 2102Ep as a reference for human embryonic stem cell research. Stem Cells 2007; 25: 437-446.

32 Brimble SN, Sherrer ES, Uhl EW, et al. The cell surface glycosphingolipids SSEA- 3 and SSEA- 4 are not essential for human ESC pluripotency. Stem Cells 2007; 25: 54-62.

33 Reubinoff BE, Pera MF, Fong C-Y, et al. Embryonic stem cell lines from human blastocysts: somatic differentiation in vitro. Nature Biotech 2000; 18: 399-404.

34 Thomson JA, Itskovitz-Eldor J, Shapiro SS, et al. Embryonic stem cell lines derived from human blastocysts. Science 1998; 282: 1145-1147.

35 Karlsson C, Bodin L, Piehl-Aulin K, et al. Tissue microarray validation: a methodologic study with special reference to lung cancer. Cancer Epidemiol Biomarkers Prev 2009; 18: 2014-2021.

36 Kao SC, Lee K, Armstrong NJ, et al. Validation of tissue microarray technology in malignant pleural mesothelioma. Pathology 2011; 43: 128-132.

37 Schmidt LH, Biesterfeld S, Kümmel A, et al. Tissue microarrays are reliable tools for the clinicopathological characterization of lung cancer tissue. Anticancer Res 2009; 29: 201-209.

38 Takahashi K, Yamanaka S. Induction of pluripotent stem cells from mouse embryonic and adult fibroblast cultures by defined factors. Cell 2006; 126: 663-676.

39 Kim J, Woo AJ, Chu J, et al. A Myc rather than core pluripotency module accounts for the shared signature of embryonic stem and cancer cells. Cell 2010; 143: 313-324.

40 Gazzeri S, Brambilla E, Caron de Fromentel C, et al. P53 genetic abnormalities and myc activation in human lung carcinoma. Int $J$ Cancer 1994; 58: 24-32.

41 Mitani S, Kamata H, Fujiwara M, et al. Analysis of c-myc DNA amplification in non-small cell lung carcinoma in comparison with small cell lung carcinoma using polymerase chain reaction. Clin Exp Med 2001; 1: 105-111.

42 Broers JL, Viallet J, Jensen SM, et al. Expression of c-myc in progenitor cells of the bronchopulmonary epithelium and in a large number of non-small cell lung cancers. Am J Respir Cell Mol Biol 1993; 9: 33-43.

43 Katagirii YU, Ohmi K, Katagiri C, et al. Prominent immunogenicity of monosialosyl galactosylgloboside, carrying a stage-specific embryonic antigen-4 (SSEA-4) epitope in the ACHN human renal tubular cell line - a simple method for producing monoclonal antibodies against detergent-insoluble microdomains/raft. Glycoconj J 2001; 18: 347-353.

44 Sullivan JP, Minna JD, Shay JW. Evidence for self-renewing lung cancer stem cells and their implications in tumor initiation, progression, and targeted therapy. Cancer Metastasis Rev 2010; 29: $61-72$. 